\title{
Prescription pattern of traditional Chinese medicine for climacteric women in Taiwan
}

\author{
Y-H. Yang, P-C. Chen, J-D. Wang, C-H. Lee and J-N. Lai* \\ Institute of Occupational Medicine and Industrial Hygiene, National Taiwan University College of \\ Public Health, Taipei; "Institute of Traditional Medicine, School of Medicine, National Yang-Ming \\ University and Department of Chinese Medicine, Taipei City Hospital, Yangming Branch, Taipei, \\ Taiwan
}

Key words: TRADITIONAL CHINESE MEDICINE, TAIWAN, CLIMACTERIC

\begin{abstract}
Background Traditional Chinese medicine (TCM) has become more popular as a therapy for symptom relief among menopause-aged women. The aim of this study was to analyze the utilization of TCM for climacteric women in Taiwan.

Methods The study analyzed frequency distributions among 19379 women aged 45-55 years, recruited from a random-sampled cohort of 200000 people from the National Health Insurance database. Data mining was conducted to explore the co-prescription patterns for finished herbal products (FHP).

Result There were 19379 women aged 45-55 years in the sample; of these, 12572 $(64.9 \%)$ utilized TCM services at least once. A total of $4078(21.0 \%)$ of the 19379 climacteric women utilized $145200(79.2 \%)$ TCM visits. Of these, $39802(21.7 \%)$ visits were because of diseases of the musculoskeletal system and connective tissue, of which more than half were treated with acupuncture and traumatology manipulative therapies. There were 28154 visits with FHP prescriptions because of non-specific symptoms and ill-defined conditions, and Jia-wei-xiao-yao-san was the most frequent formula. Nearly two-thirds of FHP contained more than two herbal formulae.

Conclusions Women of climacteric age in Taiwan utilized TCM more often than other age groups. To deal with multiple symptoms and/or diseases among climacteric women, new prescription patterns of combining two or more herbal formulae have evolved. Studies on safety issues and drug-herb interactions are warranted for future research.
\end{abstract}

\section{INTRODUCTION}

The climacteric phase is sufficiently challenging for many women for them to seek medical assistance. The findings of the Women's Health Initiative indicate that the benefits of taking estrogen plus progestin in healthy postmenopausal women are outweighed by serious risks ${ }^{1}$. Not surprisingly, alternative therapies have become increasingly popular and are quickly approaching

Correspondence: Dr J-N. Lai, Institute of Traditional Medicine, School of Medicine, National Yang-Ming University, No. I 55, Sec. 2, Linong Road, Taipei I 12, Taiwan 
hormone therapy in frequency as treatments for symptom relief among menopause-aged wo$m^{2,3}$. Unfortunately, little is known about patterns of use of traditional Chinese medicine (TCM) in conditions related to menopause, which might be one of the potential complementary and alternative medicines that can also be used in western countries. Thus, there is concern among doctors trained in conventional medicine who may treat climacteric women about drug-herb interaction and the doubtful effectiveness of TCM.

TCM, including acupuncture, traumatology manipulative therapies and Chinese herbal remedies, has been an important part of health care in Taiwan for hundreds of years and is regularly reimbursed by the National Health Insurance (NHI) since 1995. Finished herbal products (FHP) are the modern form of Chinese herbal remedies, of which single herb and herbal formulae are concentrated into granulated compounds ${ }^{4}$ and available as over-the-counter dietary supplements in the United States ${ }^{5}$. Since FHP, acupuncture and traumatology manipulative therapies are fully reimbursed under the current NHI system of Taiwan, the claim database accordingly provides a platform for the understanding of TCM utilization. The aim of our study is to analyze a random sample of this comprehensive database and determine the TCM utilization patterns for women around the climacteric age in Taiwan.

\section{MATERIALS AND METHODS}

\section{Data source}

In March of 1995, the Taiwan government implemented a NHI program which provides universal health insurance coverage; more than $97 \%$ of the whole population of Taiwan was covered by this insurance in $2002^{6}$. The computerized reimbursement database of the NHI contains medical record files, including medical care facilities and specialties, drugs and/or management for treatment, patient's gender and date of birth, date of visiting, transferred identification number, and three major diagnoses coded in the International Classification of Diseases, 9th Revision, Clinical Modification (ICD-9-CM) format. Both TCM doctors and Western medicine doctors must follow the standard diagnoses of using the ICD-9-CM coding system when claiming for reimbursement. The computerized reimbursement database of the NHI stores longitudinal data of the beneficiaries of both Western and Chinese medicines, thus providing an optimal platform for the understanding of the utilization pattern of TCM during the climacteric.

The data source included the 200000 random-sampling cohort from the NHI database 1997-2004; these were randomly selected from 23753407 people who had ever been insured under the NHI from 1995 to 2000. The sampled cohort in the present study was demonstrated to be representative of all beneficiaries including women aged between 45 and 55 years (http:// www.nhri.org.tw/nhird/file_talk/r_sing.pdf).

\section{Study subjects}

About $95 \%$ of women become menopausal between the ages of 45 and 55 years $^{7}$. Thus, this research included females aged between 45 and 55 years from the random sampling cohort as the study subjects and the age was calculated by subtracting the subject's birthday from 1st July of each year. For these subjects, we collected all records of TCM during 1997-2004 as a climacteric cohort. We considered the first diagnosis as the major diagnosis on records of visiting an outpatient department; these were coded in ICD-9 and then grouped into different broader disease categories. For example, ICD-9 codes of 710-739 were classified as diseases of the musculoskeletal system and connective tissue; codes 780-799 were grouped as symptoms, signs, and ill-defined conditions, and codes 627.0-627.9 were classified as menopausal and postmenopausal disorders.

\section{Traditional Chinese medicine}

TCM is one of the oldest healing systems in the world that has developed over the past millennia. Its practice includes FHP, acupuncture and traumatology manipulative therapies; these are reimbursed by the NHI of Taiwan. A herbal formula is composed of two or more single herbs. Traditionally, TCM doctors prescribe one herbal formula with several single herbs for each prescription to fit an individual patient's constitution. The list of reimbursed FHP in 2004 was downloaded from the website of the Bureau of NHI. The corresponding drug information about a specific mixture or name was then obtained from the Committee on Chinese Medicine and Pharmacy (CCMP) website, including the proportions of each constituent, date and period of approval as drug, code and name of manufacturer. 
Table 1 Frequency distribution of traditional Chinese medicine (TCM) visits by major disease categories (according to ICD codes) in women around climacteric age from 1997 to 2004 in Taiwan

\begin{tabular}{|c|c|c|c|c|}
\hline \multirow[b]{2}{*}{ Major disease category } & \multirow[b]{2}{*}{$\begin{array}{l}\text { ICD-9-CM } \\
\text { code range }\end{array}$} & \multicolumn{3}{|c|}{ Number of visits (\%) } \\
\hline & & $\begin{array}{c}\text { Chinese } \\
\text { herbal remedies }\end{array}$ & $\begin{array}{c}\text { Acupuncture \&o } \\
\text { traumatology }\end{array}$ & Total of TCM \\
\hline Infectious and parasitic diseases & 001-139 & $454(0.3 \%)$ & $106(0.2 \%)$ & $560(0.3 \%)$ \\
\hline Neoplasms & $140-239$ & $885(0.7 \%)$ & $116(0.2 \%)$ & $1001(0.5 \%)$ \\
\hline $\begin{array}{c}\text { Endocrine, nutritional and metabolic } \\
\text { diseases, and immunity disorders }\end{array}$ & $240-279$ & $2200(1.7 \%)$ & $342(0.7 \%)$ & $2542(1.4 \%)$ \\
\hline Mental disorders & $290-319$ & $1202(0.9 \%)$ & $211(0.4 \%)$ & $1413(0.8 \%)$ \\
\hline $\begin{array}{l}\text { Diseases of the nervous } \\
\text { system and sense organs }\end{array}$ & $320-389$ & $5780(4.4 \%)$ & $1385(2.6 \%)$ & $7165(3.9 \%)$ \\
\hline Diseases of the circulatory system & $390-459$ & $2875(2.2 \%)$ & $506(1.0 \%)$ & $3381(1.8 \%)$ \\
\hline Diseases of the respiratory system & $460-519$ & $28061(21.4 \%)$ & $2539(4.8 \%)$ & $30600(16.7 \%)$ \\
\hline Diseases of the digestive system & $520-579$ & $16986(13.0 \%)$ & $1700(3.2 \%)$ & $18686(10.2 \%)$ \\
\hline Diseases of the genitourinary system & $580-629$ & $14078(10.8 \%)$ & $1390(2.7 \%)$ & $15468(8.4 \%)$ \\
\hline $\begin{array}{l}\text { Diseases of the skin and } \\
\text { subcutaneous tissue }\end{array}$ & $680-709$ & $2893(2.2 \%)$ & $335(0.6 \%)$ & $3228(1.8 \%)$ \\
\hline $\begin{array}{l}\text { Diseases of the musculoskeletal } \\
\text { system and connective tissue }\end{array}$ & $710-739$ & $19339(14.8 \%)$ & $20463(39.0 \%)$ & $39802(21.7 \%)$ \\
\hline $\begin{array}{l}\text { Symptoms, signs and ill-defined } \\
\text { conditions }\end{array}$ & $780-799$ & $28154(21.5 \%)$ & $2057(3.9 \%)$ & $30211(16.5 \%)$ \\
\hline Injury and poisoning & $800-999$ & $6985(5.3 \%)$ & $21018(40.1 \%)$ & $28003(15.3 \%)$ \\
\hline Supplementary classification ${ }^{\dagger}$ & $\begin{array}{l}\text { V01-V82, } \\
\text { E800-E999 }\end{array}$ & $2(0.0 \%)$ & $17(0.0 \%)$ & $19(0.0 \%)$ \\
\hline Others* & & $992(0.8 \%)$ & $240(0.5 \%)$ & $1232(0.7 \%)$ \\
\hline Total & & $130886(71.4 \%)$ & $52425(28.6 \%)$ & $183311(100 \%)$ \\
\hline
\end{tabular}

*, Others include ICD-9-CM code ranges 280-289, 630-677, 740-759, 760-779 and missing/error data; ${ }^{\dagger}$, supplementary classification of factors influencing health status and contact with health service, external causes of injury and poisoning

\section{Statistical analysis}

In order to analyze the prescription patterns for different disease categories, we linked the drug registration numbers published by the Committee on Chinese Medicine and Pharmacy (CCMP) with the climacteric cohort. Frequencies and percentages of herbal formulae and/or single herbs prescribed were analyzed for each major diagnosis category. We also applied data-mining techniques to explore co-prescription patterns of medications (in FHP form) for menopausal and postmenopausal disorders. We determined, first, the support factor (the proportion of the co-prescriptions of medications $\mathrm{A}$ and $\mathrm{B}$ amongst all prescriptions), and, second, the confidence factor (the proportion of the co-prescriptions of medications $\mathrm{A}$ and $\mathrm{B}$ amongst all prescriptions containing medication A). The analysis used the SAS version 9.1 software (SAS Institute Inc. Cary, NC, USA) for data linkage and descriptive statistics of drug utilization pattern.

\section{RESULTS}

During the 8-year study period, the average annual rates of TCM utilization for women aged 45-55 years, women aged 18-44 years (younger), and women over 55 years (older) were 1.85, 1.62 , and 1.57 visits per person, respectively. Among such uses, women of climacteric age more frequently received acupuncture and traumatology manipulative therapies for musculoskeletal disorders than those of the younger and older age groups, with annual utilization rates of $0.21,0.12$ and 0.18 visits per person, accordingly.

Among the 200000 random sampling cohort, 19379 women were between the ages of 45 and 55 years and were included in the analysis. Throughout the period of 1997-2004, we found $12572(64.9 \%)$ women used TCM at least once, with a total of 183311 (average 14.6) TCM visits. Eighty-eight $(0.5 \%)$ women used only TCM without any visit to a Western medicine doctor; 
Table 2 Top ten herbal formulae prescribed by traditional Chinese medicine doctors for women around the climacteric age $(n=130886)$

\begin{tabular}{lccc}
\hline Herbal formulae & $\begin{array}{c}\text { Frequency of } \\
\text { prescriptions } n(\%)\end{array}$ & $\begin{array}{c}\text { Average daily } \\
\text { dose }(\mathrm{g})\end{array}$ & $\begin{array}{c}\text { Average duration } \\
\text { for prescriptions } \\
\text { (days) }\end{array}$ \\
\hline Jia-wei-xiao-yao-san & $11665(8.9)$ & 5.2 & 7.3 \\
Shu-jing-huo-xie-tang & $10467(8.0)$ & 5.3 & 6.2 \\
Chuan-qiong-cha-diao-san & $8052(6.2)$ & 5.1 & 5.5 \\
Ge-gen-tang & $7387(5.6)$ & 4.9 & 5.8 \\
Shao-yao-gan-cao-tang & $6359(4.9)$ & 3.7 & 6.0 \\
Ping-wei-san & $6023(4.6)$ & 3.9 & 6.0 \\
Du-huo-ji-sheng-tang & $5736(4.4)$ & 5.6 & 6.6 \\
Yin-qiao-san & $5469(4.2)$ & 5.1 & 5.3 \\
Ban-xia-xie-xin-tang & $4694(3.6)$ & 4.4 & 6.3 \\
Ma-xing-gan-shi-tang & $4464(3.4)$ & 4.5 & 5.4 \\
\hline
\end{tabular}

$4078(21.0 \%)$ of these women utilized 145200 $(79.2 \%)$ TCM visits.

Of the women visiting TCM doctors, 130886 $(71.4 \%)$ were treated with prescription of Chinese herbal remedies, while the remaining women were prescribed acupuncture and traumatology manipulative therapies, as summarized in Table 1. Most symptoms, signs and ill-defined conditions were treated with prescription of FHP, while more than half of diseases in the musculoskeletal system and most injuries were managed with acupuncture or manipulative therapies.

Table 2 presents the most common herbal formulae prescribed in the TCM visits. The top five disease categories together had a share of $81.5 \%$ of all TCM visits, with prescription of FHP as shown in Table 3 , which reveals the summation of the frequencies of most frequently prescribed herbal formulae in the top five disease categories; these appeared to follow the principles of ancient Chinese medicine in the seven most important reference books recommended by the CCMP. Further analyses using data-mining techniques identified major co-prescriptions (one-to-one association) of single herbs and herbal formulae. The most commonly prescribed herbal formula, Jia-wei-xiao-yao-san, was frequently accompanied with single herbs of Dan-shen (Salvia miltiorrhiza Bge), Suan-zao-ren (Ziziphus jujuba var. Spinosa (Bunge) Hu ex H. F. Chow), Di-gu-pi (Lycium chinense Mill), etc., or herbal formulae of Qi-ju-di-huang-wan, Liu-wei-di-huang-wan and Gan-mai-da-zao-tang. The most common prescription pattern of single herbs was Han-liancao (Eclipta prostrata Linn) and Nu-zhen-zi (Ligustrum lucidum Aiton), which were the two herbs contained in Er-Zhi-Wan. This indicates that some TCM doctors did not prescribe the
Table 3 The three most common herbal formulae prescribed by traditional Chinese medicine doctors for each of the five most common disease categories in women around climacteric age

\begin{tabular}{lr}
\hline $\begin{array}{l}\text { Disease category } \\
\text { Symptoms, signs and } \\
\text { ill-defined conditions }\end{array}$ & $\begin{array}{c}\text { Number of } \\
\text { Jia-wei-xiao-yao-san }\end{array}$ \\
Chuan-qiong-cha-diao-san & $354(21.5 \%)$ \\
Suan-zao-ren-tang & $3149(11.2 \%)$ \\
Diseases of the & $2850(10.1 \%)$ \\
$\quad$ respiratory system & $28061(21.4 \%)$ \\
Yin-qiao-san & $3861(13.8 \%)$ \\
Chuan-qiong-cha-diao-san & $3283(11.7 \%)$ \\
Ma-xing-gan-shi-tang & $3040(10.8 \%)$ \\
Diseases of musculoskeletal & $19339(14.8 \%)$ \\
$\quad$ system and connective tissue & $5854(30.3 \%)$ \\
Shu-jing-huo-xie-tang & $3831(19.8 \%)$ \\
Du-huo-ji-sheng-tang & $2467(12.8 \%)$ \\
Shao-yao-gan-cao-tang & $16986(13.0 \%)$ \\
Diseases of the digestive system & $2851(16.8 \%)$ \\
Ban-xia-xie-xin-tang & $2619(15.4 \%)$ \\
Ping-wei-san & $1629(9.6 \%)$ \\
Xiang-sha-liu-jun-zi-tang & $14078(10.8 \%)$ \\
Diseases of the & $3604(25.6 \%)$ \\
$\quad$ genitourinary system & $1204(8.6 \%)$ \\
Jia-wei-xiao-yao-san & $1008(7.2 \%)$ \\
Dang-gui-shao-yao-san & \\
Long-dan-xie-gan-tang & \\
Total &
\end{tabular}

typical formula of Er-Zhi-Wan. Instead, they prescribed these two single herbs for direct mixing or different dosage proportions of these two herbs 
in Er-Zhi-Wan to fit a particular patient's condition.

\section{DISCUSSION}

To the best of our knowledge, this study is the first of its kind using a random national-level sample to document the utilization characteristics of traditional Chinese medicine in climacteric women who sought for help in TCM therapies. Among the whole population of Taiwan, $14.1 \%$ of TCM visits were for acupuncture and traumatology manipulative therapies $^{8}$; however, they occupied $28.6 \%$ of TCM visits for women around climacteric age in our study. Among climacteric women in Taiwan, diseases of the musculoskeletal system and connective tissue were the most frequent disease category for all TCM visits, of which about two-fifths of treatment used acupuncture and traumatology manipulative therapies, as shown in Table 1.

Diseases of the musculoskeletal system and connective tissue were the major disease categories, for which the proportions of women using TCM therapies increased as age increased. Interestingly, women aged 45-55 years tended to use more non-pharmacological therapies such as acupuncture and traumatology manipulative therapies to deal with the discomfort of arthralgia and myalgia, which were common complaints during the menopausal age ${ }^{9}$. As painkillers did not seem to have substantial effectiveness and their potential toxic effects after chronic administration were major concerns ${ }^{10,11}$, we are not surprised to find an increased market for TCM.

Shu-jing-huo-xie-tang was the most commonly used herbal formula for diseases of the musculoskeletal system and connective tissue for climacteric women, as shown in Table 2. According to an ancient TCM book, Shu-jing-huo-xie-tang can dispel blood stasis and wind-dampness in the 'Channels' of the lower part of the body and the abdomen ${ }^{12}$. Despite its high frequency of prescription by TCM practitioners in Taiwan, there has not yet been any clinical trial to demonstrate its efficacy and safety.

Du-huo-ji-sheng-tang was the second most commonly uses herbal formula for diseases of the musculoskeletal system and connective tissue; it was used to treat a combination of symptoms including pain, stiffness, flaccidity and aversion to cold of the knee. Based on an ancient TCM book, these symptoms signify disharmony caused by 'Wind', 'Cold' and
'Dampness' and can be improved by Du-huo-jisheng-tang ${ }^{12}$. In fact, we have conducted a 4week outcome research, which demonstrated its effectiveness in reducing pain and stiffness of the knee joint and improving physical function for osteoarthritis $^{13}$. Future research is still needed to provide more evidence for the safety and efficacy of these medications.

Symptoms, signs and ill-defined conditions were the most frequent diagnoses in disease category for TCM visits with prescription of Chinese herbal remedies. In general, TCM practitioners treated climacteric complaints based on syndrome differentiation theory instead of making specific diagnostic codes because of holistic consideration of climacteric women with many different psychosomatic symptoms. The major drawback of this type of classification is the lack of consideration of the dynamic changes of hormonal physiology in women during this menopausal period ${ }^{14}$. For example, our previous study found that, after 12 weeks of herbal treatment, more pronounced improvements were found in perimenopausal women than postmenopausal women with similar scores on the Kupperman Index and hot flushes at the baseline examination ${ }^{15}$. Without taking different stages of ovarian insufficiency into consideration, a TCM practitioner would prescribe the same herbal formula to climacteric women with similar symptoms which might be caused by different mechanisms, resulting in unpredictable effect of TCM therapies.

Table 3 shows that Jia-wei-xiao-yao-san, Chuan-qiong-cha-diao-san and Suan-zao-ren-tang were the most common herbal formulae presented in the prescriptions for symptoms, signs and ill-defined conditions. Based on the method of data-mining, we further found that TCM doctors usually prescribed Jia-wei-xiao-yao-san together with other herbal formulae or single herbs to treat menopausal and postmenopausal disorders. Previous researches have indicated that Jia-wei-xiao-yao-san may be prescribed for relieving hot flush ${ }^{16}$ and other menopausal symptoms, including insomnia and emotional disturbance $^{15}$. Chuan-qiong-cha-diao-san is used to treat a person who has headache or migraine, which is also one of the symptoms included in the Kupperman index to estimate the severity of menopausal syndrome. Suan-zao-ren-tang is believed to be an excellent formula for insomnia, restlessness, anxiety and palpitations, which were also frequent symptoms encountered in women during the climacteric age. Thus, we concluded that the menopausal syndrome was probably 
the main cause for the higher proportion of symptoms, signs and ill-defined conditions in the diagnosis for climacteric women utilizing TCM.

FHP was invented about 40 years ago and has been utilized since then. In this study, we found that the prescription patterns of FHP by TCM doctors are different from that of decoction. Based on the TCM theory and a patient's symptom pattern, TCM doctors would prescribe a well-documented formula as the core formula and add or subtract herbs in preparing the decoction to fit an individual case. When they prescribe FHP (finished herbal products), TCM doctors can either add a single herb or several herbs into the core formula. But there is no way that they can subtract any herb or change the proportions of herbs in the finished products, because they are completely mixed together and extracted in the manufacturing process. Thus, TCM doctors can either add single herb(s) or combine other formula(e) that are manufactured into FHP to treat a patient. Because the NHI in Taiwan only reimburses FHP and not decoction, the above new prescription pattern of FHP has evolved to become a typical practice pattern of TCM doctors in Taiwan ${ }^{17}$. We found that more than $75 \%$ of prescriptions for FHP contained two or more herbal formulae. Data-mining of prescriptions for menopausal and postmenopausal disorders shows general low support for all different types of prescription pattern, which indicates a lack of consensus guidelines on prescription of FHPs for treating multiple symptoms or diseases during the climacteric age. Co-prescriptions of two or more herbal formulae such as Jia-wei-xiao-yao-san with Qiju-di-huang-wan, or with Liu-wei-di-huangwan, or with Gan-mai-da-zao-tang have not been recommended in ancient Chinese medicine textbooks, which raises concern for both their safety and effectiveness. Some adverse drug reactions, such as abdominal pain, diarrhea, pruritus, etc., were detected in a clinical trial which used three herbal formulae together for menopausal syndrome ${ }^{18}$. Thus, more outcome research is needed to test the effectiveness and safety of new prescription patterns of FHP.

Our study has four limitations. First, NHI only reimburses FHP; decoction is not reimbursed and therefore is not included and cannot be generalized as to their usage. Second, this study did not include Chinese herbal remedies purchased directly from pharmacies of traditional
Chinese medicine, nor did we include health food containing herbs. Thus, the frequency of TCM utilization might be underestimated. However, since all FHPs prescribed by TCM doctors were fully reimbursed by the NHI of Taiwan, the under-estimation might be of small magnitude. Third, we cannot come to any conclusion about the relationship between the severity of the menopausal syndrome and TCM utilization for lack of actual clinical data. Last, there is no consensus on the disease coding system for TCM. The therapeutic principle and methods of TCM are based on the result of 'syndrome differentiation', which might be the major reason for the increased frequency of ill-defined symptoms in the diagnoses of climacteric women. Future development of a more reliable coding system for TCM diagnostic classifications is important and would be a great step forward in the direction of efficient crosscultural research ${ }^{19}$.

\section{CONCLUSIONS}

The patterns of TCM utilization by women around the climacteric age are different from those in other age groups, and utilizations of acupuncture and traumatology manipulative therapies were more common in diagnoses of the musculoskeletal and connective tissue systems. Symptoms, signs and ill-defined conditions were the most frequent diagnoses for climacteric women receiving prescription of FHP from TCM doctors. Since women around the climacteric age usually suffered from multiple psychosomatic symptoms or multiple disease, these may have induced new prescription patterns of combining two or more herbal formulae for FHPs. Safety issues and drug-herb interactions should be a priority for future research and more clinical trials and outcome research are needed to assess the effectiveness of new prescription patterns in utilization of TCM.

\section{ACKNOWLEDGEMENTS}

We are grateful to the Taipei Chinese Medical Association for providing partial funding for this research, and we would also like to thank ShuChing Hsieh for her professional assistance in the statistical analysis.

\section{Conflict of interest Nil.}

Source of funding The Taipei Chinese Medical Association. 


\section{References}

1. Rossouw JE, Prentice RL, Manson JE, et al. Postmenopausal hormone therapy and risk of cardiovascular disease by age and years since menopause. JAMA 2007;297:1465-77

2. Brett KM, Keenan NL. Complementary and alternative medicine use among midlife women for reasons including menopause in the United States: 2002. Menopause 2007;14:300-7

3. Armitage GD, Suter E, Verhoef MJ, et al. Women's needs for CAM information to manage menopausal symptoms. Climacteric 2007;10: 215-24

4. Wen K-C. The turnover rate of marker constituents in Chinese herbal medicine. J Food Drug Anal 2000;8:270-7

5. Angell M, Kassirer JP. Alternative medicine - the risks of untested and unregulated remedies. $N$ Engl J Med 1998;339:839-41

6. Chou SY, Liu JT, Hammitt JK. National Health Insurance and precautionary saving: evidence from Taiwan. J Health Econ 2003;87:1873-94

7. McKinlay SM. The normal menopause transition: an overview. Maturitas 1996;23:137-45

8. Chen FP, Chen TJ, Kung YY, et al. Use frequency of traditional Chinese medicine in Taiwan. BMC Health Serv Res 2007;7:26

9. Schneider HP, Heinemann LA, Rosemeier HP, et al. The Menopause Rating Scale (MRS): comparison with Kupperman index and quality-of-life scale SF-36. Climacteric 2000;3:50-8

10. Tramer MR, Moore RA, Reynolds DJ, et al. Quantitative estimation of rare adverse events which follow a biological progression: a new model applied to chronic NSAID use. Pain 2000; 85:169-82

11. Altman R, Hochberg M, Moskowitz R, et al. Recommendations for the medical management of osteoarthritis of the hip and knee: 2000 update. American College of Rheumatology
Subcommittee on Osteoarthritis Guidelines. Arthritis Rheum 2000;43:1905-15

12. Committee on Chinese Medicine and Pharmacy. List of 100 unified formulae. [cited 19 April 2009]. Available from: http://www.ccmp.gov. tw/information/formula_search.asp? relno $=549$

13. Lai JN, Chen HJ, Chen CC, et al. Duhuo jisheng tang for treating osteoarthritis of the knee: a prospective clinical observation. Chin Med 2007;2:4

14. Hale GE, Burger HG. Hormonal changes and biomarkers in late reproductive age, menopausal transition and menopause. Best Pract Res Clin Obstet Gynaecol 2009;23:7-23

15. Lai JN, Hwang JS, Chen HJ, et al. Finished herbal product as an alternative treatment for menopausal symptoms in climacteric women. $J$ Altern Complement Med 2005;11:1075-84

16. Chen LC, Tsao YT, Yen KY, et al. A pilot study comparing the clinical effects of Jia-Wey ShiauYau San, a traditional Chinese herbal prescription, and a continuous combined hormone replacement therapy in postmenopausal women with climacteric symptoms. Maturitas 2003;44: $55-62$

17. Hsieh SC, Lai JN, Lee CF, et al. The prescribing of Chinese herbal products in Taiwan: a crosssectional analysis of the national health insurance reimbursement database. Pharmacoepidemiol Drug Saf 2008;17:609-19

18. Hsieh SC, Lai JN, Chen PC, et al. Development of active safety surveillance system for traditional Chinese medicine: an empirical study in treating climacteric women. Pharmacoepidemiol Drug Saf 2006;15:889-99

19. Melchart D, Linde K, Weidenhammer W, et al. Use of traditional drugs in a hospital of Chinese medicine in Germany. Pharmacoepidemiol Drug Saf 1999;8:115-20 